\title{
Manchmal reicht schon Pfadfinder-Tugend
}

— „Ach, wie sollen Sie mir denn helfen?", seufzte die alte Dame, die eigentlich von meiner Kollegin behandelt wird. „Sie kennen meine Krankengeschichte doch gar nicht!" Da die Kollegin zu einem Notfall aus der Sprechstunde gerufen worden war, versprach ich, mein Bestes zu versuchen. Die reizende Lady zog einen Zettel aus der Handtasche, auf dem sie die zu hohen Blutdruckwerte notiert hatte, die sie über die letzten Tage gemessen hatte. Sie berichtete auch über Kopfschmerzen, um sich dann wieder zu unterbrechen: „Aber ich kann Ihnen doch nicht alles erzählen, was in den letzten Jahren war!"

Ich beruhigte sie mit der Feststellung, dass ich das ja aus der Kartei entnehmen könnte, und fragte sie nach ihrem Blutdruckmedikament. „Das ist seit einer Woche alle, darum habe ich es nicht mehr genommen", sagte sie. „Aber dann ist doch der hohe Blut- druck kein Wunder!", rief ich. Ein echter Effekt blieb aus, sie konnte oder wollte das noch nicht so richtig verstehen. „Wirklich?“, fragte sie zweifelnd. „Aber ich habe ja auch gar keine Tabletten mehr", verfiel sie dann wieder in ihr Lamento. Ermunternd versprach ich, ihr sogleich ein Rezept auszustellen - und ich bat sie, die erste Dosis noch am selben Tag einzunehmen.

Als sie ging, war sie dankbar und erleichtert, dass ich ihr doch helfen konnte, ganz ohne ausführliche Anamnese. Wie schwer das Leben für diese nette alte Dame sein muss, wenn die simple Erlangung eines Folgerezeptes sie schon an ihre Grenzen bringt.

Dr. Frauke Höllering, Arnsberg

\section{„Mann, war das 'ne Wurst!“}

Ein dringender Anruf erreichte mich während der laufenden Sprechstunde: „Herr Doktor, mein Mann hat so schreckliche Bauchschmerzen!“, hörte ich eine aufgeregte Frauenstimme. Da mit unklaren Bauchbeschwerden nicht zu spaßen ist, ließ ich alles stehen und liegen und machte mich zügig auf den Weg zum Notfall-Hausbesuch.

Ich fand den Patienten erwartungsgemäß auf einer Couch liegend und sich den Bauch haltend. Allerdings wirkte er auf den ersten Blick nicht besonders krank. Ich machte mich also daran, den Bauch abzutasten. Doch schon nach wenigen Momenten äußerte der Patient, er müsse die Toilette aufsuchen. Von seiner
Frau wurde er ins Bad geführt. Ich muss ehrlich gestehen, dass ich ob der Verzögerung etwas unruhig wurde - schließlich hatte ich eigentlich reichlich in der Praxis zu tun.

Nach einigen eindeutigen Geräuschen aus dem Nachbarraum erschien die Ehefrau wieder bei mir mit den Worten: „Mann, war das 'ne Wurst!“ Und während sie mir weitere Einzelheiten schilderte, erschien ihr Mann mit einem erleichterten Gesichtsausdruck in der Tür. Es sei alles wieder gut, ich könne jetzt fahren.

Letztlich war ich froh, dass sich alles so einfach erledigt hatte.

Dr. Henrich Malz, Vlotho

\section{Die Diagnose ist klar: Angina italiana}

Sie war schon seit zehn Tagen krank, war aber noch nicht in die Praxis gekommen, da sie noch eine Sonde zum Mars schicken musste. So pflichtbewusst sind sie bei der Europäischen Weltraumorganisation!

Entsprechend ging es ihr dann auch: Halsschmerzen, Fieber, dicke Lymphknoten, schlechter Allgemeinzustand. „Sagen Sie mal $A !$ !, sagte ich. Auf ihr "A!" hin forderte ich scherzhaft: „E poi in italiano!“ Ich erwartete keine Reaktion, bekam aber gleich die Retourkutsche in Form eines ganz lauten und eine Oktave höheren "Aaah!" - eben eines "A!" auf italienisch. Darauf folgte noch ein schallendes Lachen.

Dr. Luise Hess, Darmstadt 